Marquette University

e-Publications@Marquette

Biomedical Engineering Faculty Research and

Publications

Biomedical Engineering, Department of

$10-1-2011$

\title{
A Compressed Sensing Algorithm for Sparse-View Pinhole Single Photon Emission Computed Tomography
}

Paul Arthur Wolf

Marquette University

Emil Y. Sidky

Taly Gilat Schmidt

Marquette University, tal.gilat-schmidt@marquette.edu

Accepted version. Published as part of the proceedings of the conference, 2011 IEEE Nuclear Science Symposium and Medical Imaging Conference (NSS/MIC), 2011: 2668-2671. DOI. (C) 2011 Institute of Electrical and Electronics Engineers (IEEE). Used with permission. 


\title{
A compressed Sensing Algorithm For Sparse-View Pinhole Single Photon Emission Computed Tomography
}

\author{
Paul A. Wolf \\ Department of Biomedical Engineering, Marquette University, \\ Milwaukee, WI \\ Emil Y. Sidky \\ Department of Radiology, University of Chicago \\ Chicago, IL \\ Taly Gilat Schmidt \\ Department of Biomedical Engineering, Marquette University, \\ Milwaukee, WI
}

\begin{abstract}
Single Photon Emission Computed Tomography (SPECT) systems are being developed with multiple cameras and without gantry rotation to provide rapid dynamic acquisitions. However, the resulting data is angularly undersampled, due to the limited number of views. We propose a novel reconstruction algorithm for sparse-view SPECT based on Compressed Sensing (CS) theory. The algorithm models Poisson noise by modifying the Iterative Hard Thresholding algorithm to minimize the Kullback-Leibler (KL) distance by gradient descent. Because the underlying objects of SPECT images are expected to be smooth, a discrete wavelet transform (DWT) using an orthogonal spline wavelet kernel is used as the sparsifying transform. Preliminary feasibility of the algorithm was tested on simulated data


of a phantom consisting of two Gaussian distributions. Single-pinhole projection data with Poisson noise were simulated at $128,60,15,10$, and 5 views over 360 degrees. Image quality was assessed using the coefficient of variation and the relative contrast between the two objects in the phantom. Overall, the results demonstrate preliminary feasibility of the proposed CS algorithm for sparse-view SPECT imaging.

\section{SECTION I.}

\section{Introduction}

Dynamic Single Photon Emission Computed Tomography (SPECT) provides information about tracer uptake and washout from a series of time-sequence images. Dynamic SPECT systems measuring time activity curves on the order of minutes have been developed. ${ }^{1,2}$ However, to adequately sample the time-activity curve of some tracers, a temporal resolution on the order of seconds is required. Stationary multiple camera systems are being developed to provide rapid dynamic acquisitions. ${ }^{3,4}$ To reduce cost, a limited number of cameras may be used, resulting in angularly undersampled data.

The image reconstruction theory of compressed sensing (CS) exploits sparsity in the object to potentially allow for a reduction in the data sampling. Thus, if some representation exists in which the coefficients of an image are sparse, the same image can be represented using less information. The object can then be accurately reconstructed from undersampled data. Reconstruction from angularly undersampled data has been recently studied for CT.5,6

We propose a novel reconstruction algorithm for sparse-view pinhole SPECT based on CS theory. The algorithm models Poisson noise statistics and uses the spline wavelet transform as the sparsifying transform to address the unique challenges of SPECT imaging.

Algorithm performance is evaluated using metrics for image fidelity and spatial accuracy. These results are compared to results obtained using Maximum-Likelihood Expectation Maximization (MLEM). 
NOT THE PUBLISHED VERSION; this is the author's final, peer-reviewed manuscript. The published version may be accessed by following the link in the citation at the bottom of the page.

\section{SECTION II.}

\section{Methods}

\section{A. The Algorithm}

CS algorithms solve a constrained optimization problem to recover the image. The $\mathrm{L}_{1}$-norm of the sparse representation is minimized and constrained by data fidelity. For example, if the data fidelity constraint is the $\mathrm{L}_{2}$-norm of the difference between the estimated and measured data, the CS optimization problem can be described as

\section{$\min .\left(\left\|\Psi_{S}\right\|_{1}\right)$ s.t. $\|\hat{H s}-y\|_{2}<\varepsilon$,}

where $H$ is the system matrix, $\hat{s}$ is the estimated image, $y$ is the measured data, $\Psi$ is a sparsifying transform, and $s$ is the true object. Data fidelity is imposed by the constraint and sparsity is enforced by the objective function. This optimization problem is solved by considering the images that satisfy the constraint, then selecting the image with the most sparse representation.

Previous CS algorithms for sparse-view tomographic reconstruction assume a piecewise constant object, using gradient magnitude as the sparsifying transform. ${ }^{6}$ In SPECT imaging, the underlying objects represent a distribution of activity, which is not necessarily piecewise constant and may be smoothly varying.

Poisson noise due to photon counting statistics can be incorporated into the CS framework by minimizing the Kullback-Leibler distance $\left(D_{K L}\right)$ to achieve data fidelity instead of the more commonly used $\mathrm{L}_{2}$ norm, which assumes Gaussian noise. In our proposed implementation, $\left(D_{K L}\right)$ is minimized by gradient descent. To account for the expected piecewise smooth nature of the tracer distribution, we propose the spline wavelet transform for the sparsifying transform, $\psi$. In our proposed algorithm, sparsity is enforced using the Iterative Hard Thresholding algorithm. ${ }^{7}$

The spline wavelet transform is characterized by having its synthesis functions be polynomial splines. If a function is piecewise smooth, the signal can be sparsely approximated by spline wavelets. The wavelet coefficients will be near zero where the 
function can be well approximated by a polynomial.8,9 Assuming underlying SPECT objects are piecewise smooth, the spline wavelet transform will operate as a sparsifying transform.

Fig. 1 shows a SPECT image of a rat-lung, the gradient magnitude image and the spline wavelet transform. The image is noisy and the underlying distribution is likely piecewise smooth. The sorted and normalized coefficients of the image, the gradient magnitude image and spline wavelet transform are shown in fig. 2 . The spline wavelet transform coefficients decay faster than the gradient magnitude coefficients, indicating that the spline wavelet transform yields a more sparse image.

Equation 2 describes the implementation of the proposed algorithm where $x$ is the sparse domain estimate of the object, $\lambda$ is the gradient descent step size, and $G_{K}$ is a nonlinear operator that retains the $\mathrm{K}$-largest coefficients setting the remaining coefficients to zero. ${ }^{7}$

$$
x^{n+1}=G_{K}\left[x^{n}-\lambda \Psi\left(\nabla D_{K L}(y, \hat{H s})\right)\right]
$$

The gradient of $\left(D_{K L}\right)$ with respect to $\hat{s}\left(\nabla D_{K L}\right)$ is

$$
\nabla_{s_{j}} D_{K L}=\sum_{m=1}^{M} H_{m, j}\left(1-y_{m} /(\hat{H s})_{m}\right), j=1, \ldots, N
$$

where $N$ is the number of voxels in the image volume and $M$ is the number of measurements. Note that this is equivalent to back projection of the parenthetical expression.

The proposed algorithm is also described by the following pseudo code. The symbol := denotes assignment, $\Psi^{-1}$ indicates the inverse sparsifying transform, $H^{T}$ indicates back projection, and $\AA$. represents the estimate of the data. 
NOT THE PUBLISHED VERSION; this is the author's final, peer-reviewed manuscript. The published version may be accessed by following the link in the citation at the bottom of the page.
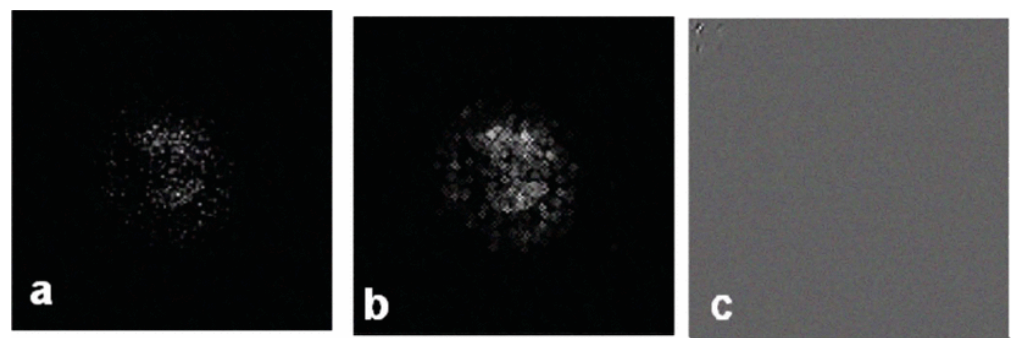

Fig. 1. (a.) rat lung image, (b.) gradient magnitude image of rat lung image, (c.) spline wavelet transform of rat lung image

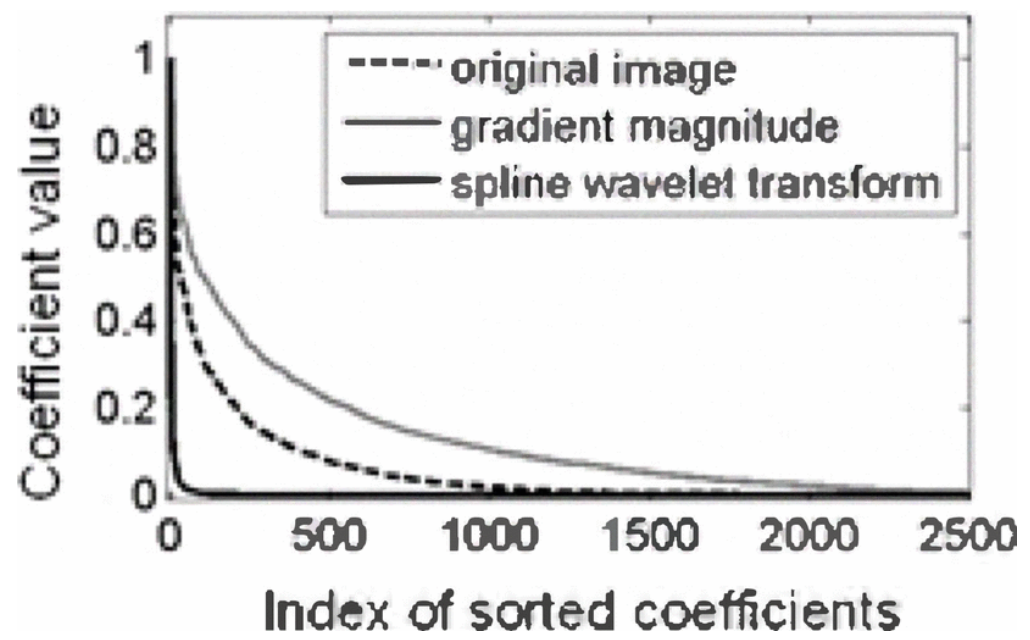

Fig. 2. A comparison of the coefficients of the rat lung image and transformed images displayed in fig. 1

\section{B. Simulation Study}

Preliminary feasibility of the algorithm was studied through simulations. The simulated object consisted of two 2D Gaussian distributions sampled onto a $128 \times 128$ pixel grid. The Gaussian objects had a standard deviation of 4 pixels and maximum values of 1 and 2. Each object was truncated at two standard deviations.

Simulated projections were generated using the system matrix, resulting in 128pixel projections at 128,60,15,10, and 5 views distributed evenly over 360 degrees. Poisson noise was added to each projection dataset such that the total number of counts remained constant as the number of views decreased.

The sparsifying transform, $\Psi$, was a 7-stage discrete wavelet transform with an orthogonal spline wavelet kernel with 5 vanishing points. The simulated object was transformed using the sparsifying transform, $\Psi$, and was determined to have 1976 nonzero coefficients. This was used as the value of the parameter $K$. 
To quantify the accuracy of the proposed algorithm, three metrics were evaluated. The coefficient of variation $(C V)$ between the estimated data and the projection data was calculated. This is a measure of image fidelity and is described by

$$
C V=\left(\sum(H \hat{s}-y)^{2}\right)^{1 / 2} / \mathrm{y}^{*} 100
$$

Quantitative accuracy was quantified using the contrast error $(C E)$,

$$
C E=\left|C_{\text {measured }}-C_{\text {true }}\right| / C_{\text {true }}
$$

$C E$ is a comparison between the reconstructed contrast and true contrast between two ROIs. $C E$ is independent of number of reconstructed counts. To quantify spatial accuracy, the scaled peak cross-correlation with the true object was used. Images were scaled using

$$
\text { ScalingFactor }=N_{\text {object }} / N_{\text {image }},(6)
$$

where $\mathrm{N}$ is the number of counts in the FOV. This metric is independent of the number of reconstructed counts. Images with a higher peak cross-correlation more accurately depict the spatial distribution of an object.

\section{SECTION III.}

\section{Results}

Fig. 3 shows the images reconstructed from 128, 60,15, 10, and 5 views using the proposed CS algorithm (a) and MLEM (b). Table 1 displays the described metrics for each algorithm and each sampling case. Fig. 4 and fig. 5 show selected profiles plotted through the center of a selection of images displayed in fig. 3.

\section{SECTION IV.}

\section{Conclusions}

Images reconstructed from ten views using both the proposed CS algorithm and MLEM depict the object contrast to < 2\% error. Spatial accuracy varied by less than $5 \%$ as the number of views decreased. 
The results of Table 1 suggest similar performance of the MLEM and proposed CS algorithm. We are currently investigating alternative sparsifying transforms and strategies to provide improved performance compared to MLEM.

128 Views

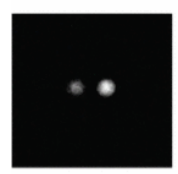

128 Views

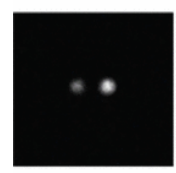

60 Views

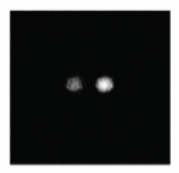

(a) Images reconstructed using the proposed CS algorithm

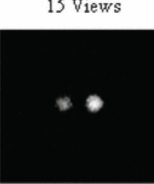

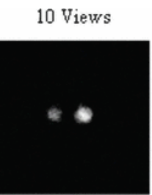

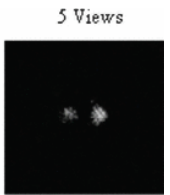

60 Views

15 Views

10 Views
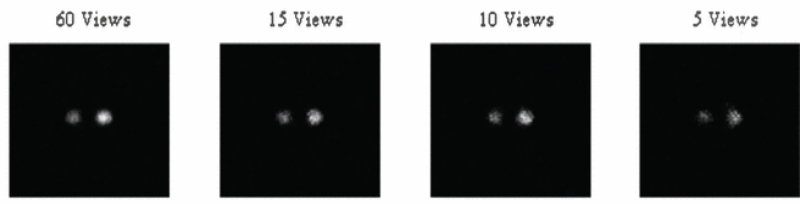

(b) Images reconstructed using MLEM

Fig. 3. Images reconstructed using (a) the proposed algorithm and (b) MLEM

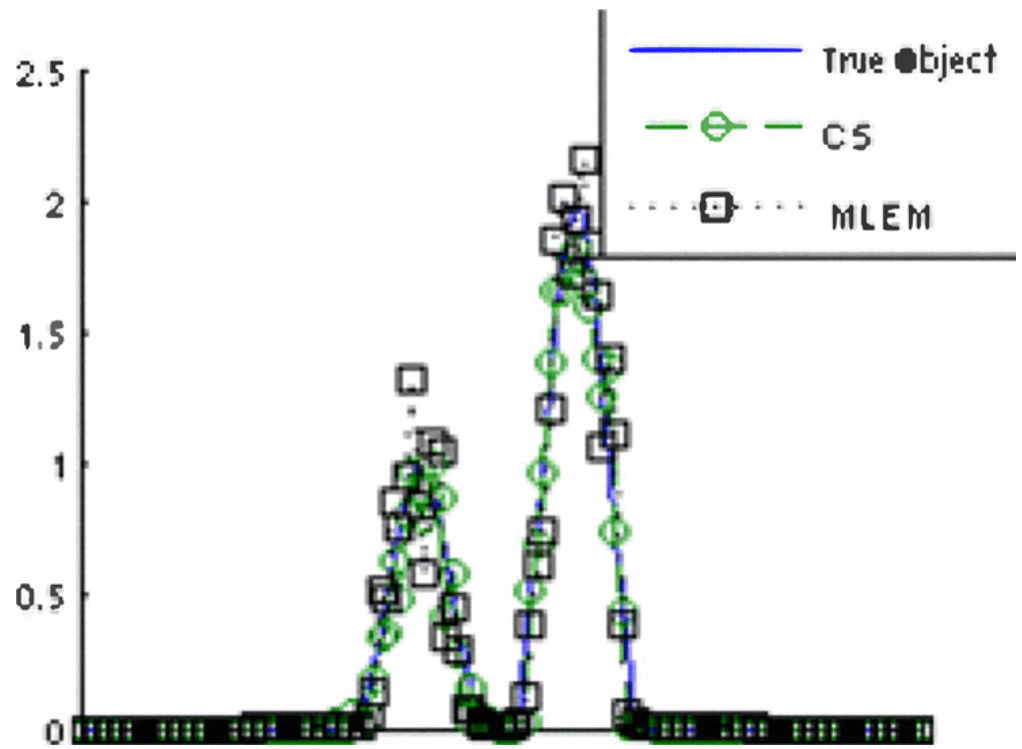

Fig. 4. Profiles through images reconstructed with 60 views 


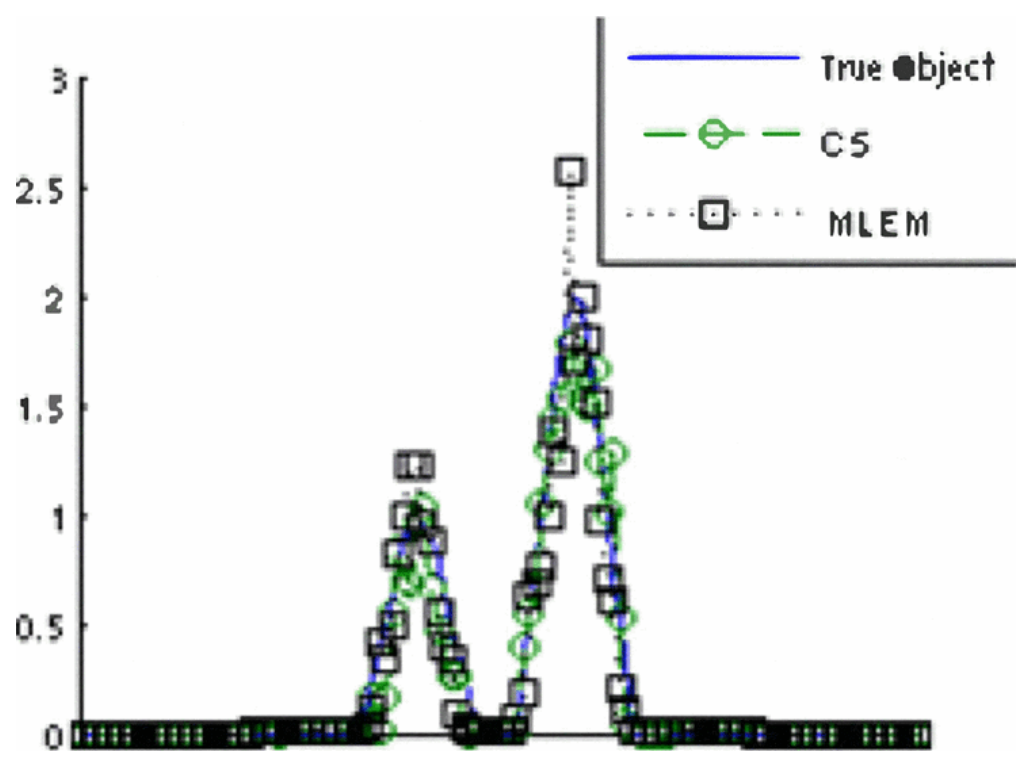

Fig. 5. Profiles through images reconstructed with 10 views

TABLE I. Image Quality Metrics for Reconstructed Images

CS

\section{Number of Views}

$\begin{array}{llllll} & \mathbf{1 2 8} & \mathbf{6 0} & \mathbf{1 5} & \mathbf{1 0} & \mathbf{5} \\ \mathrm{CV} & 3.41 & 236 & 3.03 & 2.85 & 2.11 \\ \text { CE (\%) } & 2.64 & 4.66 & 9.54 & 1.65 & 2.81\end{array}$

Peak XCorr $275.77 \quad 271.59270 .59271 .59260 .86$

MLEM

$\begin{array}{llllll}\text { CV } & 1.98 & 2.03 & 1.78 & 1.67 & 1.33 \\ \text { CE (\%) } & 1.86 & 0.42 & 1448 & 0.93 & 6.36\end{array}$

Peak Xcorr $269.6227623 \quad 276.87 \quad 271.16 \quad 261.34$

\section{References}

1G. T. Gullberg, B. W. Reutter, A. Sitek, J. S. Maltz, and T. F. Budinger, "Dynamic single photon emission computed tomography - basic principles and cardiac applications.," Physics in medicine and biology, vol. 55, no. 20, pp. R111-91, Oct. 2010.

${ }^{2} \mathrm{~A}$. Celler et al., "Preliminary results of a clinical validation of the dSPECT method for determination of renal glomerular filtration rate (GFR)," in Nuclear Science Symposium Conference Record, 2001 IEEE, 2001, vol. 2, pp. 1079-1082.

3F. J. Beekman and B. Vastenhouw, "Design and simulation of a high-resolution stationary SPECT system for small animals," Physics in Medicine and Biology, vol. 49, no. 19, pp. 4579-4592, Oct. 2004.

(Pubitemid 39378246)

IEEE Nuclear Science Symposium and Medical Imaging Conference Record, (October 2011): pg. 2668-2671. DOI. This article is C Institute of Electrical and Electronics Engineers (IEEE) and permission has been granted for this version to appear in e-Publications@Marquette. Institute of Electrical and Electronics Engineers (IEEE) does not grant permission for this article to be further copied/distributed or hosted elsewhere without the express permission from Institute of Electrical and Electronics Engineers (IEEE). 
${ }^{4}$ L. R. Furenlid et al., "FastSPECT II: A Second-Generation High-Resolution Dynamic SPECT Imager.," IEEE transactions on nuclear science, vol. 51, no. 3, pp. 631-635, Jun. 2004.

5G.-H. Chen, J. Tang, and S. Leng, "Prior image constrained compressed sensing (PICCS): A method to accurately reconstruct dynamic CT images from highly undersampled projection data sets," Medical Physics, vol. 35, no. 2, p. 660, 2008.

6E. Y. Sidky and X. Pan, "Image reconstruction in circular cone-beam computed tomography by constrained, total-variation minimization." Physics in medicine and biology, vol. 53, no. 17, pp. 4777-807, Sep. 2008.

7T. Blumensath and M. E. Davies, "Iterative hard thresholding for compressed sensing," Applied and Computational Harmonic Analysis, vol. 27, no. 3, pp. 265-274, Nov. 2009.

8M. Unser and T. Blu, "Wavelet theory demystified," Signal Processing, IEEE Transactions on, vol. 51, no. 2, pp. 470-483, 2003.

9M. Unser, "Ten good reasons for using spline wavelets," Image Processing, vol. 3169, pp. 422-431, 1997. 